\title{
Androgens and spatial ability: Failure to find a relationship between testosterone and ability measures
}

\author{
WALTER F. McKEEVER \\ Northern Arizona University, Flagstaff, Arizona \\ and \\ DEBORAH A. RICH, RICHARD A. DEYO, and ROBERT L. CONNER \\ Bowling Green State University, Bowling Green, Ohio
}

\begin{abstract}
Shute, Pellegrino, Hubert, and Reynolds (1983) found spatial ability to be positively related to androgen levels in females and negatively related to androgen levels in males. Although they intended to study the relationship of a specific steroid, testosterone (T), to spatial ability, their antibody was not specific for $\mathrm{T}$. The present study assessed the relationship of $\mathrm{T}$, specifically, to spatial and verbal abilities. Additionally, possible T-level differences between left- and righthanded subjects were assessed. Results showed females and left-handers to be inferior in spatial ability to males and right-handers, respectively, on the Stafford Identical Blocks Test. This replicates previous findings (McKeever, 1986). No other sex or handedness effects were obtained on ability measures. No handedness effect was found for $T$ levels. No relationship of $T$ to either spatial or verbal measures was found. We concluded that $T$ is not the critical androgen implicated in spatial ability by studies employing nonspecific steroid measures.
\end{abstract}

Shute, Pellegrino, Hubert, and Reynolds (1983) reported that radioimmunoassay (RIA) measures of androgens were related to spatial ability in male and female adults. The direction of the relationship was opposite for the sexes: females showed a positive and males a negative relationship between these variables. Actually, Shute et al. reported two experiments in their article.

In the first experiment, Shute et al. (1983) found no significant relationship between androgen levels and spatial ability for women, although there was a trend toward a positive relationship. The authors suggested that their failure to control for the menstrual cycle may have obscured a significant relationship. For men, a significant relationship was found for linear, quadratic, and cubic trends. Inspection of the distribution suggests that the relationship is best described as clearly negative and essentially linear.

In their second experiment, the six highest and lowest androgen-level subjects were selected from 33 new subjects. Two two-factor analyses of variance (ANOVAs), using sex and androgen level as the bases of classification, were applied to the subjects' scores on the Minnesota Paper Form Board (MPFB) and to the mean scores of two mental-rotation-type spatial tests. The results showed that

This work was supported by Grant R01 NS10214-14 from the NINCDS to the first author. The authors wish to thank Lee Meserve for the use of his RIA laboratory. Reprint requests should be sent to Walter F. McKeever, Department of Psychology, Box 15106, Northern Arizona University, Flagstaff, AZ 86011. for the MPFB there was a significant sex $\times$ androgen level interaction, which reflected a positive association of androgen level and spatial ability for females and a negative association of these factors for males.

Shute et al. (1983) used a sensitive RIA to measure plasma androgen levels, but the obtained values were too high to be indexing testosterone $(\mathrm{T})$ alone. The mean values obtained by Shute et al. for men and women were $11.2 \mathrm{ng} / \mathrm{ml}$ and $2.3 \mathrm{ng} / \mathrm{ml}$, respectively. The normal ranges of $\mathrm{T}$ for young men and women are $3.0-10.0 \mathrm{ng} / \mathrm{ml}$ and $0.2-2.0 \mathrm{ng} / \mathrm{ml}$, respectively (Funderburgh, Zipf, \& Sotos, 1983). It was apparent, therefore, that some other steroid(s) was/were bound by the particular antibody employed. For this reason, Shute et al. were careful to talk only about a relationship between spatial ability and androgens, and not about a relationship of spatial ability and $T$.

Evidence of androgen influences on spatial ability was first presented by Broverman, Broverman, Vogel, and Palmer (1964). These investigators rated, from nude photographs of the subjects, the degree of masculinity and femininity of various secondary sex characteristics such as muscle development, breast size, pubic hair distribution, shoulder width, and other characteristics. They then related these ratings to performances on "perceptual restructuring" (spatial) tasks. Their findings showed that subjects who were less strongly sex-stereotyped in secondary sex characteristics were superior in spatial ability to those who were more sex-stereotyped. This superiority of more androgynous subjects over more sex-stereotyped 
subjects was replicated by Petersen (1976), using methods similar to those of Broverman et al.

Klaiber, Broverman, and Kobayashi (1968) reported further evidence of a negative association of spatial ability and androgens in males. They measured 24-h 17-ketosteroid levels, which constitute a nonspecific androgen measure, and found that males who were lower in androgens were superior on spatial tasks to males who had higher androgen levels. Thus, the studies of Shute et al. (1983) were not the first to report associations of androgens and spatial ability. They were, however, the first to attempt to relate a specific androgen to spatial ability.

The present experiment was designed to assess the relationship of visuospatial ability to a specific androgen, namely, $\mathrm{T}$. In addition, the possibility that $\mathrm{T}$ might vary as a function of handedness was also assessed. This latter possibility was suggested by the hypothesis (Levy, 1969) that left-handers are inferior to right-handers in visuospatial ability and by our own finding (McKeever, 1986) that left-handers are inferior to right-handers on the Stafford Identical Blocks Test (SIBT), a standardized test of visuospatial ability.

\section{METHOD}

\section{Subjects}

The subjects were undergraduate students at Bowling Green State University. They were each paid \$15 to participate. There were 19 lefthanded and 22 right-handed males, and 19 left-handed and 23 righthanded females. The menstrual cycle of all female subjects was tracked prior to blood collection, and they were scheduled for blood collection at midcycle ( \pm 2 days). This was desirable, because hormone levels fluctuate systematically over the cycle and $\mathrm{T}$ tends to be highest at midcycle.

\section{Procedure}

All subjects participated in two sessions. In the first session they were given a medical screening questionnaire. Subjects who were on medications, including birth control pills, were excluded from the study, as were gay males and any subjects who acknowledged regular use of recreational drugs. Subjects having any history of neurological or serious mental disorders were also excluded. Following the completion of the screening questionnaire, the subjects took the Shipley Hartford Vocabulary Test (SHVT), a Verbal Fluency Test (VFT), which required the subject to write as many words beginning with the letter $G$ as he/she could in 2 min, and a Familial Sinistrality Questionnaire. Second sessions, all conducted at 10:00 a.m., required the subjects to complete two visuospatial tests. These were the SIBT and the MPFB. Blood samples were taken at 10:50 a.m. Blood, from a finger puncture on the nonpreferred hand, was collected in heparinized capillary tubes. The samples were spun at $2,500 \mathrm{rpm}$ for $7 \mathrm{~min}$. Plasma samples were frozen at $-23^{\circ} \mathrm{C}$ until assayed.

\section{Radioimmunoassay Measurement}

Concentrations of $\mathrm{T}$ were determined using a $\mathrm{T}-\mathrm{I}^{125}$-based commercial testosterone kit (Radioassay Systems Labs, 2015 E. University, Carson, CA 90220). The anti- $\mathrm{T}$ used is $100 \%$ reactive with $\mathrm{T}$ and has low cross-reactivity with other substances (see Funderburgh et al., 1983). Standards, samples, coated tubes and T-I ${ }^{125}$ (specific activity 1,000 $\mu \mathrm{Ci} / \mu \mathrm{g}$ ) were brought to room temperature prior to use. All reagents were used as received, with the following exception: $50 \mu \mathrm{l}$ of each T standard was diluted with $0.9 \%$ saline in the following concentrations: $1: 2$, $1: 4,1: 8$, and 1:16. Standards, diluted controls, controls, and samples were run in duplicate. Parallelism studies, in our lab and as reported by Funderburgh et al. (1983), have indicated that sample dilutions of up to $1: 16$ may be made using this kit without sacrificing sensitivity or accuracy. Hence, samples of insufficient volume to permit duplicate analyses were diluted $1: 4$ with $0.9 \%$ saline and analyzed using a standard curve with diluted standards and controls.

Twenty-five $\mu \mathrm{l}$ of each standard $(0,0.2,0.6,2.0,6.0$, and $20 \mathrm{ng} / \mathrm{ml}$ of $T$ in steroid-free human serum), control, or sample was pipetted into the anti-T coated tubes. To each tube $1 \mathrm{ml}$ of $\mathrm{T}-\mathrm{I}^{125}$ was then added, vortexed briefly, and incubated at $37^{\circ} \mathrm{C}$ for $120 \mathrm{~min}$. Following incubation, the contents of the tubes were alternately decanted and blotted on a paper towel. The tubes were transferred to a gamma counter (Micromedic Assay Compu Center) and counted for $1 \mathrm{~min}$. Results were obtained by a computer program in which log-logit transformation and weighted least squares regression analysis of data were used (see Robard, Bridson, \& Rayford, 1969). A separate standard curve was determined for each of the three runs. The $R^{2}$ values for the standard curves were excellent $(+.92,+.94,+.97)$.

\section{RESULTS}

\section{Ability Tests and Testosterone Data}

The mean scores of left- and right-handed females and males on the four ability tests and the mean group $T$ levels are shown in Table 1 . All scores were transformed to $z$ scores prior to analyses.

A series of three-factor ANOVAs was applied to various ability tests, and combinations thereof, in which the bases for classification were sex, handedness, and familial sinistrality. No significant effects were found in the analyses of the SHVT, the VFT, or the MPFB data. The effect of sex approached significance on the MPFB $(p<$ $.07)$, with males scoring higher than females. The SIBT, however, showed significant effects of $\operatorname{sex}[F(1,77)=$ $20.19, p<.0001]$, and of handedness $[F(1,77)=5.93$, $p<.0172]$. These sex and handedness effects on the SIBT replicate previous findings (McKeever, 1986). A number of verbal-spatial discrepancy scores were computed, including SIBT - SHVT, SIBT - VFT, MPFB - SHVT, MPFB - VFT, and combinations of the spatial tests minus the verbal tests and minus combinations of the verbal tests. No significant effects were found, other than those that were simply reflections of the sex and handedness effects on the SIBT.

The mean $T$ values for left-handed and right-handed females were 0.35 and $0.43 \mathrm{ng} / \mathrm{ml}$, respectively. The means for left-handed and right-handed males were 6.64 and $7.24 \mathrm{ng} / \mathrm{ml}$, respectively. A three-way ANOVA (sex, handedness, familial sinistrality) showed the obvious significant effect of sex $[F(1,77)=434.77, p<.0001]$. The

Table 1

Means and SDs of Left-Handed (LH) and Right-Handed (RH) Females and Males for SIBT, MPFB, VFT, SHVT, and T Measures

\begin{tabular}{|c|c|c|c|c|c|c|c|c|}
\hline & \multicolumn{2}{|c|}{ LH Females } & \multicolumn{2}{|c|}{ RH Females } & \multicolumn{2}{|c|}{ LH Males } & \multicolumn{2}{|c|}{ RH Males } \\
\hline & Mean & SD & Mean & SD & Mean & SD & Mean & SD \\
\hline & 12.84 & 7.43 & 18.00 & 2.86 & 2168 & 6.77 & 24.18 & 6.26 \\
\hline MPFB & & & & & & & & \\
\hline VFT & & 5.5 & & & & & & \\
\hline SHVT & & 2.8 & & 2. & & & & 3.25 \\
\hline & 0.35 & 0.32 & 0.43 & 0.71 & 6.64 & 2.23 & 7.24 & 1.53 \\
\hline
\end{tabular}


Table 2

Correlation of Testosterone with SIBT, MPFB, VFT, and SHVT Measures for Sex-Handedness Groups

\begin{tabular}{|c|c|c|c|c|c|c|c|c|}
\hline & \multicolumn{2}{|c|}{ LH Females } & \multicolumn{2}{|c|}{ RH Females } & \multicolumn{2}{|c|}{ LH Males } & \multicolumn{2}{|c|}{ RH Males } \\
\hline & $r$ & $P$ & $r$ & $P$ & $r$ & $P$ & $r$ & $P$ \\
\hline SIBT & -.12 & .63 & -.14 & .53 & +.27 & .25 & -.02 & .91 \\
\hline MPFB & -.30 & .21 & -.01 & .96 & +.23 & .33 & -.21 & .34 \\
\hline VFT & +.14 & .57 & +.05 & .82 & +.20 & .42 & -.20 & .37 \\
\hline SHVT & -.09 & .71 & +.01 & .96 & +.17 & .47 & +.20 & .36 \\
\hline
\end{tabular}

effect of handedness was nonsignificant $(p<.23)$, as was the effect of familial sinistrality $(p<.40)$. No interaction was significant.

\section{Relationships of Testosterone to Ability Test Performances}

Table 2 presents the correlations of $\mathrm{T}$ with various ability measures for each sex-handedness group. It can be seen that none of the correlations was significant. Correlations (not shown in the table) were also computed for male and female subjects separately. Again, no significant correlations were obtained.

\section{DISCUSSION}

With respect to the major question addressed by this study, namely, whether $\mathbf{T}$ is related to spatial ability or to spatial-verbal-ability discrepancy scores, the data are clearly negative. Circulating $\mathrm{T}$ levels were not correlated with such ability measures in males nor females. Furthermore, our failure to find a relationship in females cannot be attributed to uncharted variations in menstrual cycles, since we controlled for this factor. It should be noted that our findings suggest that it would be difficult to find a relationship of $T$ to other variables in college-age females simply because of the rather extreme range restriction of $T$ in this population.

Although these findings might appear inconsistent with those of Shute et al. (1983), one should recall that despite their original intention to measure $T$ levels, they actually measured a nonspecific androgen complex. Gordon and Lee (1986) recently measured T levels and correlated these with various verbal- and spatial-ability measures in 32 men who were tested in two sessions, 1 week apart. The subjects took all tests on both occasions. In the initial session, $T$ was found to correlate significantly and positively $(+.54)$ with a particular spatial test (orientation) of the test battery used by Gordon and Lee. In the second session, however, the correlation was nonsignificant $(-.02)$. Thus, no replicable relationship of $T$ to spatial ability could be demonstrated, and it is notable that the significant relationship obtained in the first session was in an opposite direction from that found by Shute and her colleagues.
In short, neither the present study nor that of Gordon and Lee (1986), both of which measured $\mathrm{T}$ specifically, found any relationship to ability measures. It seems possible, therefore, that some gonadal steroid other than $\mathrm{T}$ could be responsible for the positive findings of Shute et al. (1983) and Klaiber et al. (1968), both of whom employed a nonspecific steroid measure.

The failure to find differences in $\mathrm{T}$ levels between handedness groups is not surprising, and the differential spatial ability of handedness groups, replicated in the present study, cannot be due to T-level differences. It is also of interest that performances on the MPFB, the test Shute et al. (1983) found most related to their androgen measure, were not significantly influenced by either sex or handedness. It would seem advisable for studies attempting to find relationships between specific androgens and spatial ability to include both mental-rotation tasks, such as the SIBT and the orientation task of Gordon and Lee (1986), and spatial-relations tasks, such as the MPFB.

Finally, the negative results of the present experiment and of Gordon and Lee (1986) should not lead us to abandon the effort to identify possible specific steroid influences on ability levels or on patterns of abilities. The fact that two studies using nonspecific androgen measures found such measures related to spatial ability argues for further efforts to identify the specific steroid(s) accounting for these relationships.

\section{REFERENCES}

Broverman, D. M., Broverman, I. K., Vogel, W., \& Palmer, R. D. (1964). The automatization cognitive style and physical development. Child Development, 35, 1343-1359.

FUNDERBURG, L. J., ZIPF, W. B., \& Sotos, J. F. (1983). Direct measurement of testosterone in a pediatric center, with use of a radioimmunoassay kit and unextracted serum. Clinical Chemistry, 29, 1796-1798.

GoRDON, H. W., \& LEE, P. A. (1986). A relationship between gonadetropins and visuo-spatial function. Neuropsychologia, 24, 563-576.

Klaiber, E. L., Broverman, D. M., \& Kobayashi, Y. (1968). The automatization cognitive style, androgens, and monoamine oxidase. Psychopharmacologia, 11, 320-333.

LEvY, J. (1969). Possible basis for the evolution of lateral specialization of the human brain. Nature: Lord, 224, 614-615.

MCKeEVER, W. F. (1986). The influences of handedness, sex, familial sinistrality, and androgyny of language laterality, verbal ability, and spatial ability. Cortex, 22, 521-537.

Petersen, A. C. (1976). Physical androgyny and cognitive functioning in adolescence. Developmental Psychology, 12, 524-533.

RodBARD, D., BRIDSON, W., \& RAYFORD, P. L. (1969). Rapid calculation of radioimmunoassay results. Journal of Laboratory \& Clinical Medicine, 79, 770-781.

Shute, V. J., Pellegrino, J. W., Hubert, L., \& Reynolds, R. W. (1983). The relationship between androgen levels and human spatial abilities. Bulletin of the Psychonomic Society, 21, 465-468.

(Manuscript received for publication April 20, 1987.) 